Virginia Commonwealth University VCU Scholars Compass

2012

\title{
Two-step thermal quenching of photoluminescence in Zn-doped GaN
}

Michael A. Reshchikov

VirginiaCommonwealth University, mreshchi@vcu.edu

Follow this and additional works at: http://scholarscompass.vcu.edu/phys_pubs

Part of the Physics Commons

Reshchikov, M.A. Two-step thermal quenching of photoluminescence in Zn-doped GaN. Physical Review B, 85, 245203 (2012). Copyright (C) 2012 American Physical Society.

\section{Downloaded from}

http://scholarscompass.vcu.edu/phys_pubs/53

This Article is brought to you for free and open access by the Dept. of Physics at VCU Scholars Compass. It has been accepted for inclusion in Physics Publications by an authorized administrator of VCU Scholars Compass. For more information, please contact libcompass@vcu.edu. 


\title{
Two-step thermal quenching of photoluminescence in $\mathrm{Zn-doped} \mathrm{GaN}$
}

\author{
Michael A. Reshchikov* \\ Department of Physics, Virginia Commonwealth University, Richmond, Virginia 23284-2000, USA
}

(Received 18 April 2012; published 4 June 2012)

\begin{abstract}
We observed tunable two-step thermal quenching of photoluminescence in high-resistivity $\mathrm{Zn}$-doped GaN. The characteristic temperatures of the first and second steps increase with increasing excitation intensity. The effect is explained within a phenomenological model involving shallow donors, nonradiative deep donors, and two types of acceptors.
\end{abstract}

DOI: 10.1103/PhysRevB.85.245203

PACS number(s): 78.55.Cr, 71.55.Eq

\section{INTRODUCTION}

Variation of temperature is widely used in photoluminescence (PL) studies to explain effects related to point defects in semiconductors and to determine some of their important properties. In conductive semiconductors, the behavior of the defect-related PL upon changing temperature is relatively well understood. For example, in conductive $n$-type GaN, most PL bands are related to different types of acceptors. ${ }^{1}$ These PL bands are quenched above a characteristic temperature $T_{0}$. When the logarithm of PL intensity is plotted as a function of inverse temperature (the Arrhenius plot), the slope of this dependence at $T>T_{0}$ reveals the acceptor's ionization energy. Both $T_{0}$ and the slope of the thermal quenching increase linearly with increasing the acceptor's ionization energy. ${ }^{1}$ However, in high-resistivity semiconductors the behavior of PL is much less studied and not well understood.

Recently, we observed tunable and abrupt thermal quenching of the blue luminescence (BL) band in high-resistivity Zn-doped GaN. ${ }^{2}$ The BL band in undoped and $\mathrm{Zn}$-doped GaN has a maximum at $2.9 \mathrm{eV}$, the zero-phonon line at $3.10 \mathrm{eV}$, and a characteristic fine structure at low temperature. ${ }^{1}$ In conductive $n$-type GaN samples, the thermal quenching of this band begins at $T_{0}=200 \mathrm{~K}$, which is independent of the excitation intensity. The activation energy of this quenching is about $0.35 \mathrm{eV}$, in agreement with the ionization energy of the $\mathrm{Zn}_{\mathrm{Ga}}$ acceptor. However, in high-resistivity Zn-doped GaN, the BL intensity drops abruptly at a characteristic temperature $T^{*}$, and this temperature increases with increasing excitation intensity; i.e., the quenching is tunable. The unusual PL quenching behavior was explained by a model of a high-resistivity semiconductor with three types of defects: a shallow donor, the $\mathrm{Zn}_{\mathrm{Ga}}$ acceptor, and a deep donor. ${ }^{2}$ The latter is responsible for nonradiative recombination. The tunable and abrupt thermal quenching of the Zn-related BL band in high-resistivity GaN samples is caused by a sudden transition of the system from a population inversion at $T<T^{*}$ to a quasiequilibrium population at $T>$ $T^{*}$. This transition is initiated by thermal emission of holes from the $\mathrm{Zn}_{\mathrm{Ga}}$ acceptor and their consequent recombination with electrons via deep nonradiative defects. ${ }^{2}$

Closer inspection of the PL data from a number of $\mathrm{Zn}$-doped $\mathrm{GaN}$ samples revealed the following interesting effect. While in nearly all the samples only one step of abrupt quenching of PL was observed, in one of the samples (sample s560) two steps could be observed in the temperature dependence of the BL band: a small step that begins at $T_{1} \approx 130 \mathrm{~K}$ and a large step at $T_{2} \approx 200 \mathrm{~K}$. This sample was also special for another reason: the intensity of the ultraviolet luminescence (UVL) band for it was the highest among eight GaN:Zn samples which demonstrated the tunable and abrupt quenching of the BL band.

In this work, we provide the evidence that the additional step in the BL band quenching is related to the emission of holes from the shallow acceptor to the valence band. Remarkably, positions of both steps could be tuned by excitation intensity. While tunable quenching of PL in phosphors has been reported in the past,,$^{3-5}$ this is the first observation of the tunable two-step quenching of defect-related PL in semiconductors. The PL data have been explained within the phenomenological approach developed earlier, ${ }^{2}$ with the difference that the shallow acceptor with the activation energy of $0.2 \mathrm{eV}$ was included additionally in the analysis.

This paper comprises the following sections: Experimental details and results are presented in Secs. II and III, respectively; a phenomenological model for PL in a high-resistivity semiconductor with two types of acceptors is presented in Sec. IV; comparison of the theory with the experimental data and discussion are presented in Sec. V; and the main conclusions are given in Sec. VI.

\section{EXPERIMENTAL}

We studied a number of high-resistivity Zn-doped GaN layers with thicknesses between 3 and $10 \mu \mathrm{m}$ grown by the hydride vapor phase epitaxy (HVPE) method on $c$-plane sapphire. ${ }^{2}$ Eight out of nine samples exhibited dramatic PL quenching; namely, the BL band intensity dropped abruptly at a characteristic temperature $T^{*}$, and this temperature increased with increasing excitation intensity. The only sample that exhibited an additional stepwise quenching at lower temperatures was sample s560, the detailed study of which is reported in this work. Concentration of $\mathrm{Zn}$ is unknown for this sample, and the Hall effect results are inconclusive because of high resistivity and less then perfect contacts. However, with the hot probe method we observed very weak $p$-type conductivity at room temperature.

Steady-state PL was excited with a continuous-wave $\mathrm{He}$ $\mathrm{Cd}$ laser $(50 \mathrm{~mW}$ at $325 \mathrm{~nm})$, and the $\mathrm{PL}$ signal was dispersed by a $0.3 \mathrm{~m}$ monochromator and detected by a cooled photomultiplier tube. By using neutral density filters and an unfocused laser beam with a diameter of $4 \mathrm{~mm}$, the excitation power density $\left(P_{\text {exc }}\right)$ was varied from $2 \times 10^{-7}$ to $0.3 \mathrm{~W} / \mathrm{cm}^{2}$. A closed-cycle optical cryostat was employed for the temperature range of $15-330 \mathrm{~K}$. The PL spectra were corrected for the response of the optical system. The absolute 

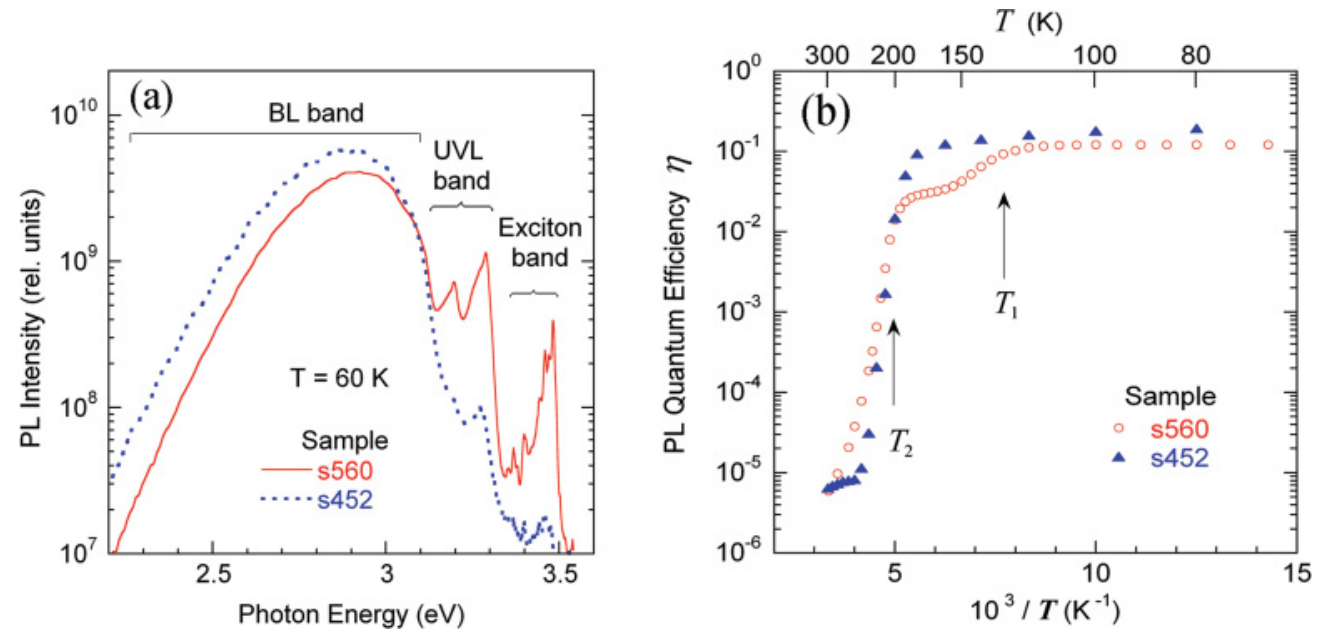

FIG. 1. (Color online) PL spectra and thermal quenching behavior of the BL band in two high-resistivity Zn-doped GaN samples (s452 and s560) at $P_{\mathrm{exc}}=0.3 \mathrm{~W} / \mathrm{cm}^{2}$. (a) PL spectra at $60 \mathrm{~K}$. (b) Temperature dependence of the BL quantum efficiency.

internal quantum efficiency of PL, $\eta$, is defined as $\eta=I^{\mathrm{PL}} / G$, where $I^{\mathrm{PL}}\left[\mathrm{cm}^{-3} \mathrm{~s}^{-1}\right]$ is the PL intensity from a particular defect (from $\mathrm{Zn}_{\mathrm{Ga}}$ in the case of the BL band), and $G$ is the concentration of electron-hole pairs created by incident laser light per unit time in the same volume. To estimate $\eta$, we compared the integrated intensity of the BL band in the studied sample with PL intensity obtained from the standard GaN and bulk $\mathrm{ZnO}$ samples previously calibrated, ${ }^{2,6}$ and measured under identical conditions and with known $P_{\text {exc }}$.

\section{RESULTS}

Figure 1(a) compares the PL spectra from two highresistivity GaN:Zn samples at $60 \mathrm{~K}$. Three main bands can be resolved: exciton emission at photon energies between 3.3 and $3.5 \mathrm{eV}$, the UVL band in the region from 3.1 to $3.3 \mathrm{eV}$, and the BL band between 2.2 and $3.1 \mathrm{eV}$. The temperature of $60 \mathrm{~K}$ was chosen to better demonstrate the contribution from the UVL band, which is hidden under the exciton wing at lower temperatures in some samples. While the intensity of the BL band in these two samples is about the same, the intensity of the UVL band in sample s560 is an order of magnitude higher, which indicates a higher concentration of shallow acceptors in this sample. As can be seen from Table I in Ref. 2, the relative intensity and the absolute internal quantum efficiency of the UVL band were the highest for this sample among eight high-resistivity GaN:Zn samples. Remarkably, only for this sample we did observe two steps in the quenching of the BL band. The first (small) step in the BL band quenching occurs at $T_{1} \approx 130 \mathrm{~K}$, and the second (large and abrupt) step at $T_{2} \approx$ $200 \mathrm{~K}$ [Fig. 1(b)]. Below, we will analyze the PL data for sample s560 in more detail.

Evolution of the PL spectrum with increasing temperature is shown in Fig. 2. Figure 2(a) shows the whole spectrum at selected temperatures between 25 and $300 \mathrm{~K}$, whereas Figs. 2(b) and 2(c) show the excitonic and UVL band regions in more detail at temperatures between 15 and $50 \mathrm{~K}$. As can be seen from Fig. 2(a), the thermal quenching of the BL band starts above $100 \mathrm{~K}$, simultaneously with the quenching of the UVL band. The quenching of the BL band slows down at temperatures between 125 and $175 \mathrm{~K}$, and a very abrupt quenching is observed at higher temperatures.

As can be seen from Fig. 2(b), the excitonic emission at low temperatures is dominated by a line at $3.461 \mathrm{eV}$, which is attributed to an $A$ exciton bound to the neutral $\mathrm{Zn}_{\mathrm{Ga}}$ acceptor $\left(\mathrm{Zn} X_{\mathrm{A}}\right)$. It is followed by an LO phonon replica at $3.370 \mathrm{eV}$. Much weaker peaks at 3.473 and $3.484 \mathrm{eV}$ are attributed to an exciton bound to a neutral shallow acceptor $\left(A X_{\mathrm{A}}\right)$ and the free $A$ exciton $\left(X_{A}\right)$, respectively. Due to strain in the GaN layer, all the peaks are shifted by about $6 \mathrm{meV}$ to higher energy as compared to their positions in relaxed GaN. ${ }^{1}$ The quantum efficiency of the exciton emission (integrated over the range from 3.3 to $3.5 \mathrm{eV}$ ) increased from 0.002 to 0.1 with increasing excitation power density from $3 \times 10^{-5}$ to $0.3 \mathrm{~W} / \mathrm{cm}^{2}$ at $15 \mathrm{~K}$. With increasing temperature from 15 to $100 \mathrm{~K}$, the acceptor-bound exciton emission is quenched and disappears [Figs. 2(a) and 2(b)]. At higher temperatures, the excitonic emission is governed by annihilation of free excitons.

The UVL band includes the zero-phonon line at $\sim 3.27 \mathrm{eV}$ and an LO phonon replica at $3.18 \mathrm{eV}$. Its absolute internal quantum efficiency in the studied sample is about 0.01 at $15 \mathrm{~K}$. The UVL band consists of two sets of lines, which can be resolved by varying the temperature from 15 to $50 \mathrm{~K}$ [Fig. 2(c)]. One set (the strongest peak at $3.269 \mathrm{eV}$ and its LO phonon replica at $3.177 \mathrm{eV}$ ) dominates at low temperature, and it is attributed to donor-acceptor pair (DAP) transitions involving a shallow donor (presumably oxygen in a nitrogen site, $\mathrm{O}_{\mathrm{N}}$ ) and an unidentified shallow acceptor. Another set (main peak at $3.289 \mathrm{eV}$ and its $\mathrm{LO}$ phonon replica at $3.197 \mathrm{eV}$ ) is attributed to transitions from the conduction band to the same shallow acceptor, commonly labeled as the $e A$ transition. With increasing temperature from 15 to $50 \mathrm{~K}$, the DAP emission gives way to the $e A$ emission because electrons from the shallow donor are thermally emitted to the conduction band. Separation between the $e A$ and DAP peaks is $20 \mathrm{meV}$. One of the reasons why this value is smaller than the ionization energy of the $\mathrm{O}_{\mathrm{N}}$ donor in very pure $\mathrm{GaN}$ samples $(33 \mathrm{meV})^{1}$ is that the DAP peaks shift to higher photon energies with increasing excitation intensity, as well as for the samples with high concentration of donors and acceptors. 

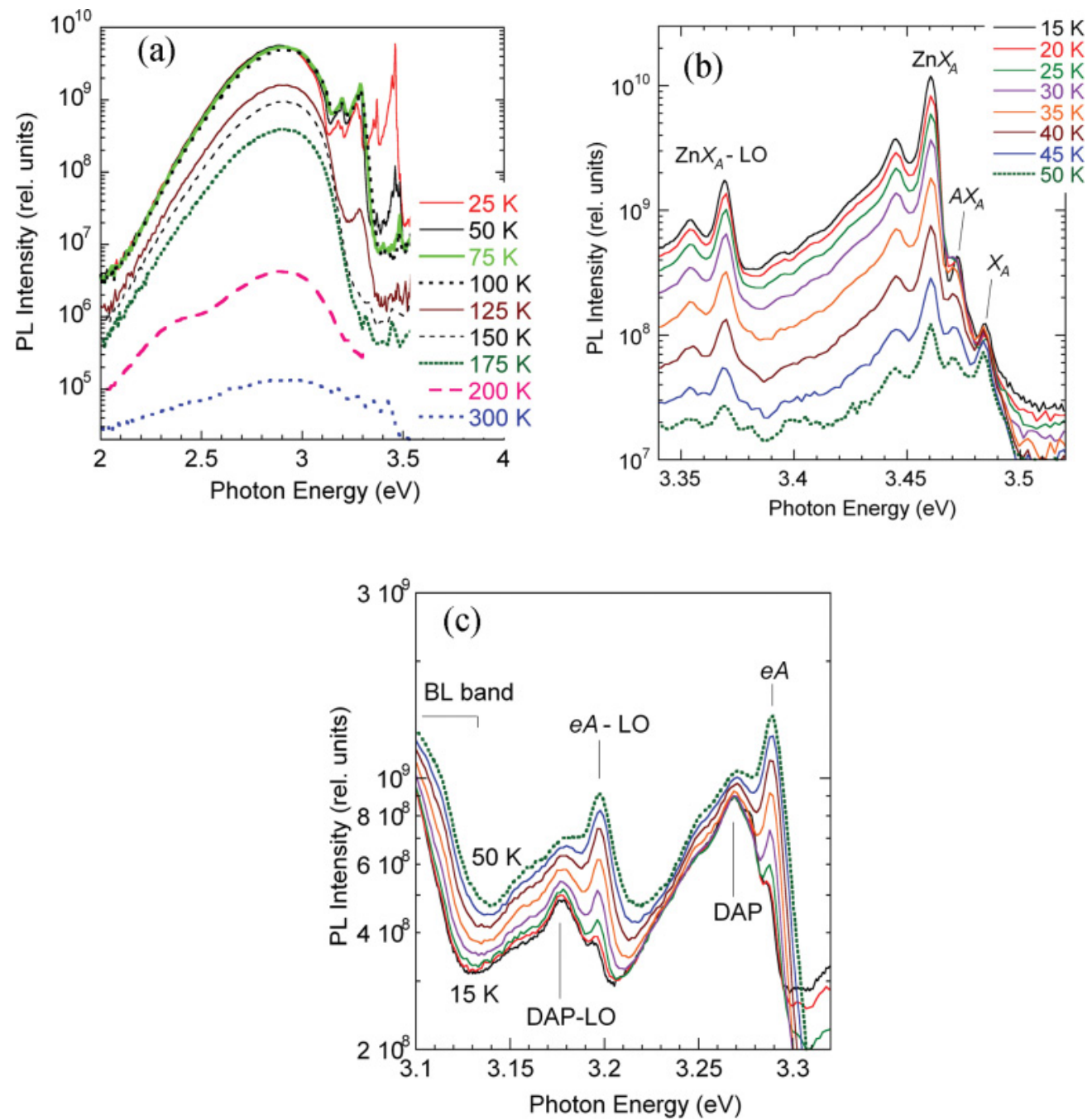

FIG. 2. (Color online) Evolution of PL spectrum with temperature for sample s560. $P_{\mathrm{exc}}=0.011 \mathrm{~W} / \mathrm{cm}^{2}$. (a) Whole spectrum, (b) excitonic region, (c) UVL band region.

The integrated intensity of the UVL band slowly increases with increasing temperature from 50 to $100 \mathrm{~K}$, which is mostly due to the increasing contribution of its $e A$ component (Fig. 3). The quenching of this band begins at temperature $T_{1} \approx 130 \mathrm{~K}$ (Fig. 3). From the Arrhenius plot, the activation energy of this quenching is estimated as $\sim 0.2 \mathrm{eV}$, which agrees with the energy level of the shallow acceptor in GaN. ${ }^{1}$ In contrast to the behavior of PL in conductive $n$-type GaN, where the quenching of one PL band causes an increase in the intensities of other PL bands, ${ }^{1,7,8}$ we observed a significant decrease in the intensities of the BL band and excitonic emission in the temperature region where the UVL band is quenched (Fig. 3). This is the first step in quenching of the BL band. It is a step, because with increasing temperature from 150 to $180 \mathrm{~K}$, the slope of the quenching in the Arrhenius plot decreases substantially.

At $T_{2} \approx 200 \mathrm{~K}$, the BL band is quenched very abruptly, and we call this the second step of the quenching (Fig. 3). The UVL and excitonic emission bands cannot be resolved at these temperatures anymore. A remarkable observation is that the values of $T_{1}$ and $T_{2}$ gradually shift to lower temperatures with decreasing excitation intensity (Fig. 4).

Following the conventions adopted in Refs. 2 and 8, the characteristic temperatures $T_{1}$ and $T_{2}$ will be defined as temperatures where the temperature-independent parts and the parts with the largest slope in the Arrhenius plot cross when extrapolated. When the values of $10^{3} / T_{1}$ and $10^{3} / T_{2}$ for the first and second steps of the BL band quenching are plotted as a logarithmic function of either the excitation intensity or the electron-hole pair generation rate $G$, a linear dependence can be noticed for both steps (Fig. 5). This dependence can be described with the following expression:

$$
T_{i}=\frac{E_{A i}}{k \ln \left(B_{i} / G\right)},
$$

where $E_{A i}$ is the ionization energy for the shallow acceptor $(i=1)$ and $\mathrm{Zn}_{\mathrm{Ga}}$ acceptor $(i=2), k$ is Boltzmann's constant, and $B_{i}$ is a constant for each acceptor. For the first step, we find $E_{A 1}=200 \pm 10 \mathrm{meV}$ and $B_{1} \approx 10^{11} N_{v} \mathrm{~cm}^{-3} \mathrm{~s}^{-1}$, where $N_{v}$ is the effective density of states in the valence band. For the 


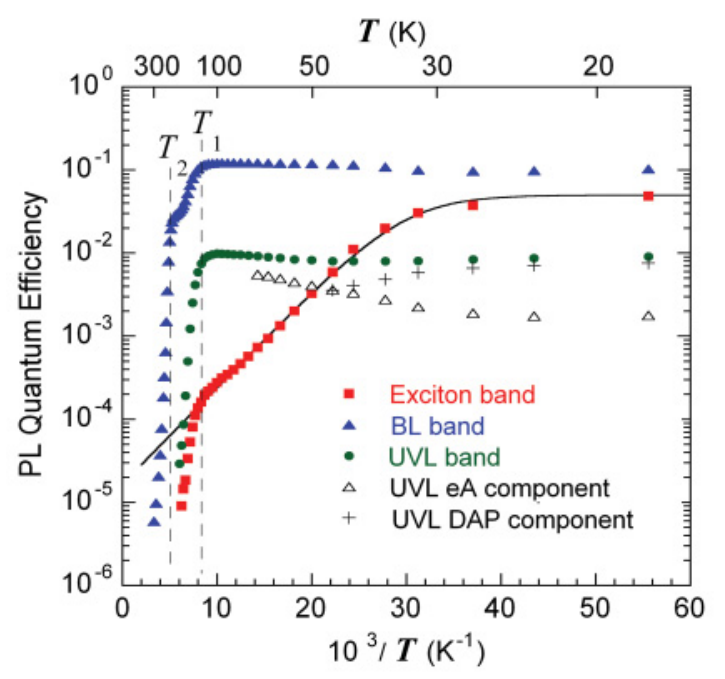

FIG. 3. (Color online) Temperature dependence of the quantum efficiency for different PL bands in sample s560. $P_{\text {exc }}=0.3 \mathrm{~W} / \mathrm{cm}^{2}$. Solid curve is calculated by using Eq. (1) in Ref. 2 with $E_{A}=23 \mathrm{meV}$.

second step, $E_{A 2}=350 \pm 20 \mathrm{meV}$ and $B_{2} \approx 10^{12} N_{v} \mathrm{~cm}^{-3}$ $\mathrm{s}^{-1}$. Note that a weak temperature dependence for $N_{v}$ can be ignored in the first approximation.

The tunable and abrupt thermal quenching of the BL band in $\mathrm{Zn}$-doped $\mathrm{GaN}$ has been explained previously by a sudden, thermally driven, redirection of recombination currents from radiative to nonradiative channels. ${ }^{2}$ However, the two-step tunable quenching of PL has never been reported before. Below, we will explain this unusual behavior of PL using a simple phenomenological model.

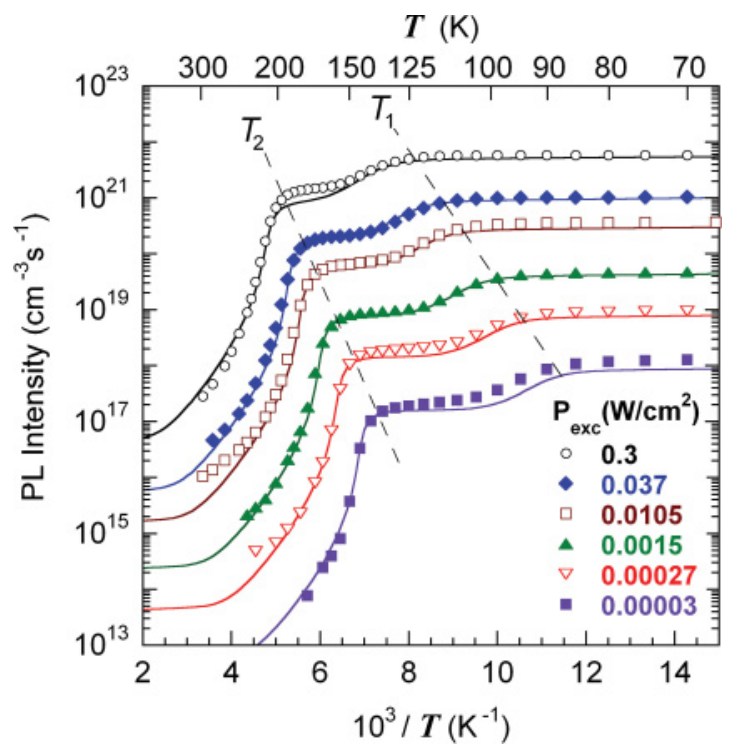

FIG. 4. (Color online) Temperature dependence of PL intensity for the BL band in Zn-doped $\mathrm{GaN}$ (sample s560) at selected excitation intensities. Solid curves are from the numerical solution of Eqs. (2)(8) with the parameters listed as Set 1 in Table I. Dashed lines show schematically the shift of the characteristic temperatures $T_{1}$ and $T_{2}$ with decreasing excitation intensity.

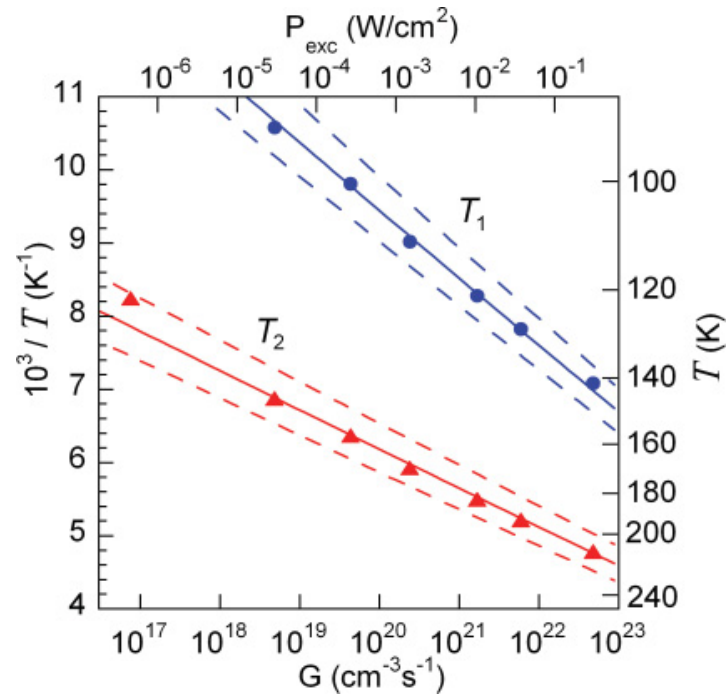

FIG. 5. (Color online) Dependence of $10^{3} / T_{1}$ and $10^{3} / T_{2}$ on excitation intensity for the BL band in sample s560. Solid lines are calculated by using Eqs. (26) and (31) with the following parameters: $E_{A 1}=200 \mathrm{meV}$ and $B_{1}=10^{11} N_{v} \mathrm{~cm}^{-3} \mathrm{~s}^{-1}$ for the UVL band, and $E_{A 2}=350 \mathrm{meV}$ and $B_{2}=1.3 \times 10^{12} N_{v} \mathrm{~cm}^{-3} \mathrm{~s}^{-1}$ for the BL band. Dashed lines are calculated by using the same values for coefficients $B_{1}$ and $B_{2}$ and the following activation energies: $E_{A 1}=190$ and $210 \mathrm{meV}$ for the UVL band (higher and lower lines, respectively, for the dependence labeled $T_{1}$ ), and $E_{A 2}=330$ and $370 \mathrm{meV}$ for the BL band (higher and lower lines, respectively, for the dependence labeled $\left.T_{2}\right)$.

\section{MODEL}

Let us consider a semiconductor containing four types of point defects: a shallow donor $D$, a shallow acceptor $A_{1}$, a deeper acceptor $A_{2}$, and a deep nonradiative donor $S$ with total concentrations $N_{D}, N_{A 1}, N_{A 2}$, and $N_{S}$, respectively (Fig. 6). The ionization energies of the shallow donor and two acceptors are $E_{D}, E_{A 1}$, and $E_{A 2}$, whereas nonradiative centers $S$ have deep donor level(s), so that the carriers cannot escape from them at the temperatures used in our experiment. In Zn-doped $\mathrm{GaN}$, shallow donors are $\mathrm{O}_{\mathrm{N}}$ centers $\left(E_{D} \approx 20-30 \mathrm{meV}\right)$ introduced unintentionally; shallow acceptors have uncertain origin $\left(E_{A 1} \approx 200 \mathrm{meV}\right)$; and deeper acceptors are $\mathrm{Zn}_{\mathrm{Ga}}$ centers $\left(E_{A 2} \approx 350 \mathrm{meV}\right)$. As for the nonradiative centers, it is predicted that point defects with deep levels often have very strong electron-phonon coupling and large lattice relaxation, so that transitions of electrons (or holes) from the conduction band to the defect and from the defect to the valence band are both nonradiative and cause emission of multiple phonons. ${ }^{9}$ Very little is known about the nonradiative point defects in $\mathrm{GaN}$. However, it is expected that these may be deep donors when the sample is doped with acceptors and deep acceptors when it is doped with donors, due to the self-compensation effect. ${ }^{10}$

The main transitions of electrons and holes under the conditions of PL are shown schematically in Fig. 6. Illumination by a laser with above-band-gap energy and excitation power density $P_{\text {exc }}$ creates electron-hole pairs with the rate $G$. These charge carriers may form excitons or be captured by point defects. In the first approximation, we can ignore the exciton 


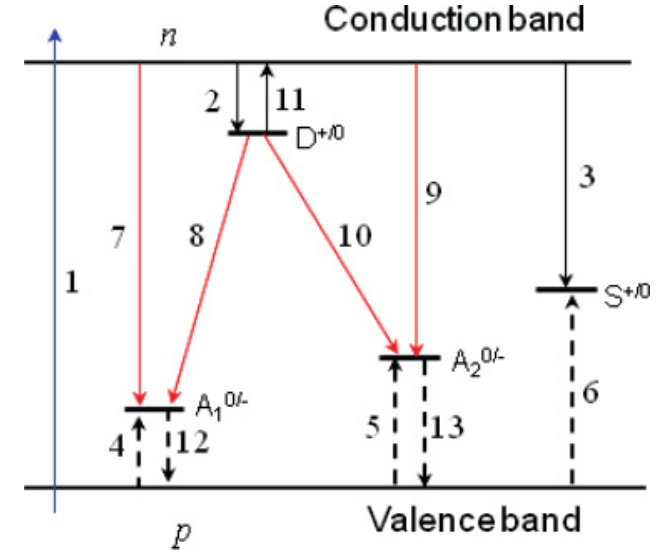

FIG. 6. (Color online) Band diagram and main transitions for a semiconductor with a shallow donor $(D)$, shallow and deeper acceptors $\left(A_{1}\right.$ and $\left.A_{2}\right)$, and a nonradiative deep donor $(S)$. Transitions of electrons and holes are shown with solid and dashed arrows, respectively. 1 , excitation of the electron-hole pair; 2 and 3 , nonradiative capture of electrons by $D$ and $S$ centers, respectively; 4, 5, and 6, nonradiative capture of holes by $A_{1}, A_{2}$, and $S$ centers, respectively; 7 and $8, e A$ and DAP emission including the $A_{1}$ acceptor; 9 and $10, e A$ and DAP emission including the $A_{2}$ acceptor; 11 , thermal emission of electrons from $D$ centers to the conduction band; 12 and 13, thermal emission of holes from $A_{1}$ and $A_{2}$ centers to the valence band.

recombination because its contribution to the total PL intensity at temperatures above $80 \mathrm{~K}$ (where the two-step quenching is observed) is small. It is small at $P_{\text {exc }}=0.3 \mathrm{~W} / \mathrm{cm}^{2}$ (Fig. 3), and it only decreases at lower excitation intensities.

At low temperature $(T<20 \mathrm{~K})$, photogenerated electrons are quickly captured by positively charged donors, and holes are captured by negatively charged acceptors and nonradiative centers. All these capture transitions are fast (on a time scale of $\sim 10^{-12}$ to $10^{-10} \mathrm{~s}$ ) and are nonradiative. Subsequently, a tunnel transition of the electrons between spatially separated shallow donors and acceptors is possible, with the transition probability depending on the sizes of the electron and hole wave functions and the separations between shallow donors and acceptors. There is a random distribution of the separations, and thus the characteristic time of the transition varies widely for different donor-acceptor pairs. For simplicity, we introduce the average probability for such transitions, characterized by a capture coefficient $C_{\text {DA }}$ (different for different types of acceptors). The probability for a photogenerated hole to be captured by a shallow donor or for an electron to be captured directly by a neutral acceptor is typically much lower than that for their recombination via the DAP mechanism, at least at low temperatures when the concentration of free electrons is much lower than the concentration of electrons bound to shallow donors.

At elevated temperatures $(T>30 \mathrm{~K})$, the concentration of free electrons increases due to thermal emission of electrons from the shallow donors. At these temperatures, transitions from the conduction band to the acceptor ( $e A$ transitions) start competing with the DAP transitions. Note that the $e A$ and DAP transitions are often unresolved in the PL spectrum, especially in the case of broad PL bands, because $E_{D}$ is much smaller than the PL band width. However, in the case of the UVL band, we were able to resolve these transitions [Fig. 2(c)] and analyze the temperature dependencies of these two components separately.

Transition rates will be traditionally described as the product of the concentrations of available carriers and available empty sites, with a constant factor representing a capture coefficient. ${ }^{11,12}$ We can then write the following rate equations for the steady-state conditions:

$$
\begin{aligned}
& G=C_{n S} N_{S}^{+} n+C_{n D} N_{D}^{+} n+C_{n A 1} N_{A 1}^{0} n+C_{n A 2} N_{A 2}^{0} n \\
& -Q_{D} N_{D}^{0} \\
& G=C_{p S} N_{S}^{0} p+C_{p A 1} N_{A 1}^{-} p+C_{p A 2} N_{A 2}^{-} p-Q_{A 1} N_{A 1}^{0} \\
& -Q_{A 2} N_{A 2}^{0} \text {, } \\
& C_{n D} N_{D}^{+} n-Q_{D} N_{D}^{0}=C_{D A 1} N_{D}^{0} N_{A 1}^{0}+C_{D A 2} N_{D}^{0} N_{A 2}^{0}, \\
& C_{n S} N_{S}^{+} n=C_{p S} N_{S}^{0} p \\
& C_{D A 1} N_{D}^{0} N_{A 1}^{0}+C_{n A 1} N_{A 1}^{0} n=C_{p A 1} N_{A 1}^{-} p-Q_{A 1} N_{A 1}^{0}, \\
& C_{D A 2} N_{D}^{0} N_{A 2}^{0}+C_{n A 2} N_{A 2}^{0} n=C_{p A 2} N_{A 2}^{-} p-Q_{A 2} N_{A 2}^{0}, \\
& p+N_{S}^{+}+N_{D}^{+}=n+N_{A 1}^{-}+N_{A 2}^{-} \text {. }
\end{aligned}
$$

Here, Eqs. (2) and (3) describe the balance of electrons and holes in the conduction and valence bands, respectively; Eqs. (4) and (5) describe balance of electrons at shallow donors $D$ and nonradiative centers $S$; Eqs. (6) and (7) describe balance of holes at shallow and deeper acceptors, $A_{1}$ and $A_{2}$; and Eq. (8) describes the conservation of charge in a semiconductor. In these equations, $n$ and $p$ are concentrations of free electrons and holes; $C_{n D}, C_{n A 1}, C_{n A 2}$, and $C_{n S}$ are electron-capture coefficients for the shallow donor, two acceptors and nonradiative defect, respectively; $C_{p A 1}, C_{p A 2}$, and $C_{p S}$ are hole-capture coefficients for two acceptors and nonradiative defect, respectively; and $C_{D A 1}$ and $C_{D A 2}$ are the effective coefficients for DAP recombination involving the $A_{1}$ and $A_{2}$ acceptors, respectively. Variables $N_{D}^{+}, N_{D}^{0}, N_{S}^{+}, N_{S}^{0}$, $N_{A 1}^{0}, N_{A 1}^{-}, N_{A 2}^{0}$, and $N_{A 2}^{-}$represent the concentrations of the $D$, $S, A_{1}$, and $A_{2}$ centers in different charge states. Finally, $Q_{D}$, $Q_{A 1}$, and $Q_{A 2}$ are electron and hole emission coefficients. These coefficients can be found from detailed balance as

$$
\begin{aligned}
Q_{D} & =C_{n D} N_{c} g^{-1} \exp \left(-E_{D} / k T\right), \\
Q_{A 1} & =C_{p A 1} N_{v} g^{-1} \exp \left(-E_{A 1} / k T\right),
\end{aligned}
$$

and

$$
Q_{A 2}=C_{p A 2} N_{v} g^{-1} \exp \left(-E_{A 2} / k T\right),
$$

where $N_{c}$ and $N_{v}$ are the effective densities of states in the conduction and valence bands, respectively, and $g$ is the degeneracy factor for the donor and acceptor levels (assumed to be equal to 2 for all).

The fit of the temperature dependencies of the BL band intensity with numeric solutions of Eqs. (2)-(8) is shown in Fig. 4 with solid curves. The two steps in the PL quenching and their shift with changing excitation intensity are reproduced. 
TABLE I. Parameters of the model used in calculations.

\begin{tabular}{lcr}
\hline \hline Parameter & Set 1 & \multicolumn{1}{c}{ Set 2} \\
\hline$N_{A 1}, \mathrm{~cm}^{-3}$ & $1.56 \times 10^{17}$ & $2 \times 10^{17}$ \\
$N_{A 2}, \mathrm{~cm}^{-3}$ & $2.7 \times 10^{17}$ & $10^{18}$ \\
$N_{S}, \mathrm{~cm}^{-3}$ & $2.5 \times 10^{17}$ & $5 \times 10^{17}$ \\
$N_{D}, \mathrm{~cm}^{-3}$ & $1.7 \times 10^{17}$ & $2.5 \times 10^{17}$ \\
$C_{p A 1}, \mathrm{~cm}^{3} / \mathrm{s}$ & $7 \times 10^{-8}$ & $10^{-6}$ \\
$C_{p A 2}, \mathrm{~cm}^{3} / \mathrm{s}$ & $7 \times 10^{-7}$ & $10^{-6}$ \\
$C_{p S}, \mathrm{~cm}^{3} / \mathrm{s}$ & $1.2 \times 10^{-6}$ & $10^{-6}$ \\
$C_{n S}, \mathrm{~cm}^{3} / \mathrm{s}$ & $10^{-8}$ & $10^{-7}$ \\
$C_{n D}, \mathrm{~cm}^{3} / \mathrm{s}$ & $10^{-8}$ & $10^{-8}$ \\
$C_{n A 1}, \mathrm{~cm}^{3} / \mathrm{s}$ & $2 \times 10^{-13}$ & $10^{-13}$ \\
$C_{n A 2}, \mathrm{~cm}^{3} / \mathrm{s}$ & $4 \times 10^{-13}$ & $10^{-13}$ \\
$C_{D A 1}, \mathrm{~cm}^{3} / \mathrm{s}$ & $1.5 \times 10^{-14}$ & $10^{-13}$ \\
$C_{D A 2}, \mathrm{~cm}^{3} / \mathrm{s}$ & $4 \times 10^{-13}$ & $10^{-13}$ \\
$E_{A 1}, \mathrm{meV}^{-13}$ & 190 & 200 \\
$E_{A 2}, \mathrm{meV}$ & 360 & 350 \\
$E_{D}, \mathrm{meV}$ & 5 & 20 \\
\hline \hline
\end{tabular}

The parameters used in this fit are listed in Table I as Set 1. In Sec. V, we will discuss how unique and reasonable the model parameters are. However, in order to obtain simple analytical expressions, we will assume in this section that the capture parameters of two acceptors are identical: $C_{n A 1}=C_{n A 2}=$ $C_{n A}, C_{p A 1}=C_{p A 2}=C_{p A}$, and $C_{D A 1}=C_{D A 2}=C_{D A}$. Model parameters for this case are listed in Table I as Set 2.

Below, we will obtain expressions for the most important and interesting case of a $p$-type semiconductor-moderately conductive or high-resistivity-for which $N_{A 1}+N_{A 2}>N_{D}+$ $N_{S}$ and $N_{A 2}>N_{A 1}$. In such a semiconductor, the Fermi level is close to the $A_{2}$ level in dark. Four characteristic regions can be selected in the temperature dependence, where the PL intensity is constant or varies slowly with temperature. We will label these regions with Roman numerals I, II, III, and IV and analyze them below. Main features of another interesting case (a high-resistivity $n$-type semiconductor) will be also briefly reviewed.

\section{A. Low temperatures}

In a $p$-type semiconductor with $N_{A 1}+N_{A 2}>N_{D}+N_{S}$, shallow donors and nonradiative deep donors lack electrons in dark. When illuminated with above-band-gap light at low temperature, the shallow donors remain almost completely empty $\left(N_{D}^{+} \approx N_{D}\right)$, while the nonradiative defects become partially filled with electrons. Their population with electrons is independent of the excitation intensity in steady state; however, the steady-state equilibrium is achieved faster for higher excitation intensities. The total concentration of holes bound to two types of acceptors increases by nearly the same amount when the excitation light is turned on.

The numeric solutions of Eqs. (2)-(8) with parameters from Set 2 in Table I and $G=10^{20} \mathrm{~cm}^{-3} \mathrm{~s}^{-1}$ for concentrations of defects in different charge states and for PL intensity associated with the shallow and deeper acceptors are shown in Figs. 7 and 8 , respectively.

At very low temperatures (region I, $T<20 \mathrm{~K}$ ), thermal emission of electrons from the shallow donors to the conduction band and holes from the acceptors to the valence

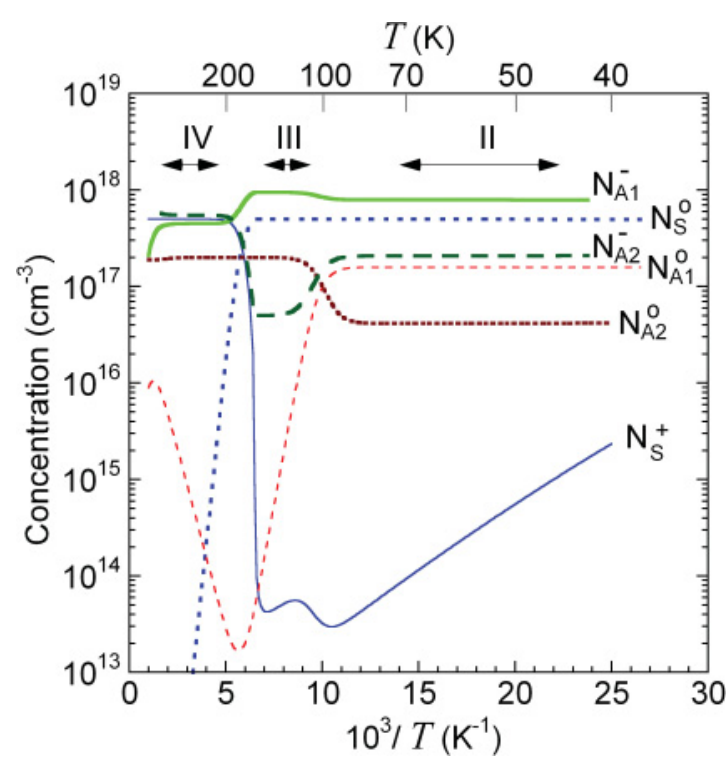

FIG. 7. (Color online) Calculated dependencies of concentrations of $S$ centers and two acceptors in different charge states. Numerical calculations with Eqs. (2)-(8) use parameters from Set 2 in Table I and $G=10^{20} \mathrm{~cm}^{-3} \mathrm{~s}^{-1}$. Roman numerals on top indicate temperature regions discussed in the text.

band can be ignored $\left(Q_{D}=Q_{A 1}=Q_{A 2}=0\right)$. In this case, the concentrations of free electrons and holes can be ignored in Eq. (8), so that $N_{D}^{+} \approx N_{D}, N_{A 1}^{-}+N_{A 2}^{-} \approx N_{D}+N_{S}^{+}$, and the steady-state concentration of positively charged $S$ centers

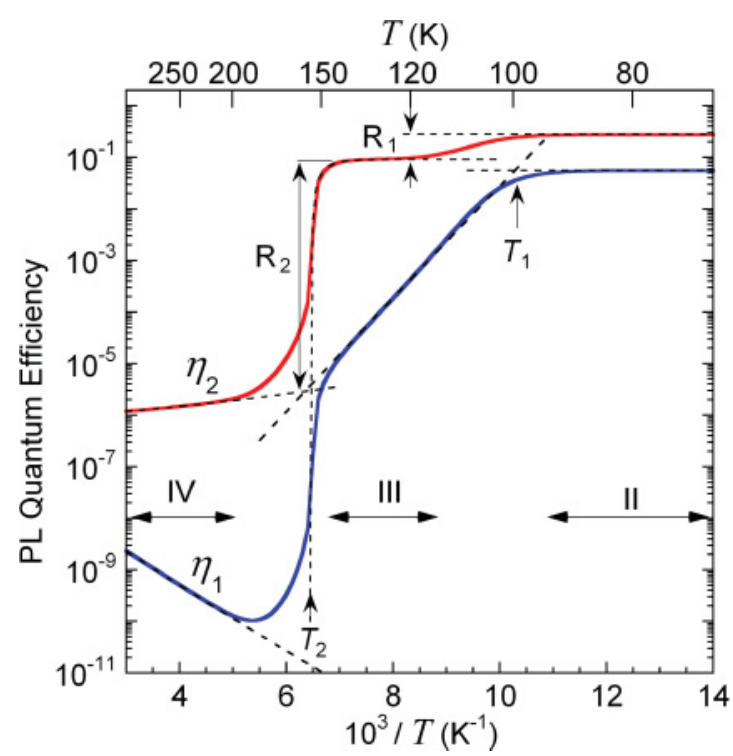

FIG. 8. (Color online) Temperature dependence of the quantum efficiency of PL associated with acceptors $A_{1}\left(\eta_{1}\right)$ and $A_{2}\left(\eta_{2}\right)$. Solid lines are numerical solutions of Eqs. (2)-(8). Labels $\eta_{1}$ and $\eta_{2}$ indicate the PL via the shallow acceptor and the $\mathrm{Zn}_{\mathrm{Ga}}$ acceptor, respectively. Dashed lines are approximate solutions with Eqs. (19) and (20) in region II, Eqs. (24) and (25) in region III, and Eqs. (33) and (34) in region IV, for $\eta_{1}$ and $\eta_{2}$, respectively. $G=10^{20} \mathrm{~cm}^{-3} \mathrm{~s}^{-1}$ and other parameters are given in Table I as Set 2. 
can be found as

$$
N_{S}^{+} \approx N_{1}=\frac{N_{D}}{2}\left[\sqrt{(1+\delta)^{2}+4 \delta \frac{N_{S}}{N_{D}}}-(1+\delta)\right],
$$

where $\delta=\left(C_{n D} C_{p S}\right) /\left(C_{n S} C_{p A}\right)$. The $S$ centers become partially filled with electrons in steady state $\left(N_{S}^{0}=N_{S}-N_{1}\right)$, and the total concentration of holes bound to two acceptors increases by the same amount $\left(N_{1}\right)$ under illumination. The relative occupation of the acceptors with electrons is the same when their capture parameters are identical:

$$
\frac{N_{A 1}^{-}}{N_{A 1}}=\frac{N_{A 2}^{-}}{N_{A 2}}=\frac{N_{D}+N_{1}}{N_{A 1}+N_{A 2}} .
$$

Contribution of the DAP-type transitions to the acceptorrelated PL significantly exceeds contribution from the $e A$-type transitions, and the PL quantum efficiency for two acceptors in region I $\left(\eta_{1, I}\right.$ and $\left.\eta_{2, I}\right)$ is independent of temperature:

$$
\eta_{1, I}=\frac{C_{p A} N_{A 1}\left(N_{D}+N_{1}\right)}{\left(N_{A 1}+N_{A 2}\right)\left[C_{p A}\left(N_{D}+N_{1}\right)+C_{p S}\left(N_{S}-N_{1}\right)\right]}
$$

and

$$
\eta_{2, I}=\eta_{1, I} \frac{N_{A 2}}{N_{A 1}}
$$

For parameters in Set $2, N_{1} \approx 4 \times 10^{16} \mathrm{~cm}^{-3}, \eta_{1, I} \approx 0.06$, and $\eta_{2, I} \approx 0.30$.

With increasing temperature (above $T \approx 30 \mathrm{~K}$ ), electrons, after being captured by shallow donors, may escape to the conduction band instead of being recombined with holes via the DAP mechanism. This leads to a gradual increase of $n$ and therefore an increase of the $e A$ luminescence intensity with the activation energy of $E_{D}$. Simultaneously, $N_{S}^{+}$decreases with the same activation energy, and the $S$ centers become almost completely filled with electrons $\left(N_{S}^{0} \approx N_{S}\right)$ due to increased concentration of electrons in the conduction band and their fast capture by $S$ centers. Temperature region II $(50 \mathrm{~K}<$ $T<90 \mathrm{~K}$ ) can be defined as the region where the emission of electrons from the shallow donors to the conduction band is significant so that $C_{n D} N_{D}^{+} n \approx Q_{D} N_{D}^{0}$ and $N_{S}^{0} \approx N_{S}$, but the emission of holes from acceptors to the valence band is still negligible. The situation is identical to the case of one acceptor at $T<100 \mathrm{~K},{ }^{2}$ if we combine two acceptors into one; i.e., $N_{A 1}+N_{A 2}=N_{A}$. A bottleneck for recombination of photogenerated carriers is created at these temperatures in the $S$ channel, because there are insufficient holes in the valence band that can be captured by the $S$ center and that can open the path for electron recombination via this center. The charge neutrality equation and the relative occupation of the acceptors with holes become

$$
N_{A 1}^{-}+N_{A 2}^{-} \approx N_{D}
$$

and

$$
\frac{N_{A 1}^{-}}{N_{A 1}}=\frac{N_{A 2}^{-}}{N_{A 2}}=\frac{N_{D}}{N_{A 1}+N_{A 2}},
$$

respectively. The concentration of free holes in this temperature region is independent of temperature and consists mostly of optically generated holes:

$$
p=p_{I I}=\frac{G}{C_{p S} N_{S}+C_{p A} N_{D}} .
$$

The acceptor-related PL quantum efficiency ( $e A$ plus DAP) for two acceptors in the temperature region II $\left(\eta_{I I}\right)$ is independent of temperature and has the following components:

$$
\eta_{1, I I}=\frac{C_{p A} N_{A 1} N_{D}}{\left(N_{A 1}+N_{A 2}\right)\left(C_{p S} N_{S}+C_{p A} N_{D}\right)}
$$

and

$$
\eta_{2, I I}=\eta_{1, I I} \frac{N_{A 2}}{N_{A 1}} .
$$

For parameters in Set $2, \eta_{1, I I} \approx 0.056$ and $\eta_{2, I I} \approx 0.28$. The quantum efficiency of PL via acceptors does not change much during the transition from region I to region II, provided that $C_{n D}<C_{n S}$.

\section{B. First step in thermal quenching of photoluminescence}

At higher temperatures (transition from region II to region III begins at $T \approx 100 \mathrm{~K}$ ), the thermal emission of holes from the shallow acceptor level to the valence band cannot be ignored. This emission causes the first step in the quenching of PL from both the shallow and deeper acceptors. A relatively small first step in the quenching of PL from the deeper acceptor is observed when $N_{A 1}<N_{D}$. We will consider this case below.

Analysis of Eqs. (2)-(8) shows that holes emitted from the shallow acceptor are captured essentially by the deeper acceptor, so that concentration of negatively charged deeper acceptors decreases from $N_{A 2}^{-}=N_{D} N_{A 2} /\left(N_{A 1}+N_{A 2}\right)$ to $N_{A 2}^{-}=N_{D}-N_{A 1}$, whereas $S$ centers remain almost completely filled with electrons; i.e., $N_{S}^{0} \approx N_{S}$. The concentration of holes in the valence band after the first step saturates at

$$
p=p_{I I I}=\frac{G}{C_{p S} N_{S}+C_{p A}\left(N_{D}-N_{A 1}\right)} .
$$

The concentration of holes bound to the shallow acceptor decreases exponentially in region III as

$$
N_{A 1}^{0}=\frac{C_{p A} N_{A 1}}{Q_{A 1}} p=p_{I I I} \frac{g N_{A 1}}{N_{v}} \exp \left(\frac{E_{A 1}}{k T}\right) .
$$

From Eq. (7) with $Q_{A 2} N_{A 2}^{0}=0$, we can obtain an expression for $N_{D}^{0}$ in region III:

$$
N_{D}^{0}=\frac{C_{p A}\left(N_{D}-N_{A 1}\right) p_{I I I}}{\left(C_{n A} \frac{N_{c}}{g N_{D}} e^{-E_{D} / k T}+C_{D A}\right)\left(N_{A 1}+N_{A 2}-N_{D}\right)} .
$$

Then the quantum efficiency of PL via the shallow acceptor can be found as

$$
\begin{aligned}
\eta_{1, I I I}= & \frac{C_{p A} N_{A 1}\left(N_{D}-N_{A 1}\right) G}{\left(N_{A 1}+N_{A 2}-N_{D}\right)\left[C_{p S} N_{S}+C_{p A}\left(N_{D}-N_{A 1}\right)\right]^{2}} \\
& \times \frac{g}{N_{v}} \exp \left(\frac{E_{A 1}}{k T}\right) .
\end{aligned}
$$

The quantum efficiency of PL related to the shallow acceptor decreases exponentially as $\eta_{1, I I I} \propto \exp \left(E_{A 1} / k T\right)$; i.e., we expect to see in the Arrhenius plot the quenching of PL related to the shallow acceptor with the activation energy 
$E_{A 1}$, similar to the quenching of PL in a conductive $n$-type semiconductor. $^{2,7}$ The quantum efficiency of PL related to the deeper acceptor decreases in the transition from region II to region III and saturates at a new level

$$
\eta_{2, I I I}=\frac{C_{p A} N_{A 2}^{-} p}{G}=\frac{C_{p A}\left(N_{D}-N_{A 1}\right)}{C_{p S} N_{S}+C_{p A}\left(N_{D}-N_{A 1}\right)} .
$$

For parameters in Set $2, \eta_{2, I I I} \approx 0.09$ in region III. Thus, both PL bands associated with the shallow and deeper acceptors are quenched at a certain characteristic temperature $T_{1}$, with the difference that this quenching stops for PL via the deeper acceptor and continues for the shallow one.

The characteristic temperature $T_{1}$ can be defined as the temperature at which the exponential dependence of $\eta_{1, I I I}$ in region III extrapolated to lower temperatures crosses its constant value in region II, $\eta_{1, I I}$; i.e., $\eta_{1, I I I}\left(T=T_{1}\right)=\eta_{1, I I}$. By equating Eqs. (19) and (24), we find

$$
T_{1}=\frac{E_{A 1}}{k \ln \left(B_{1} / G\right)}
$$

with

$$
B_{1}=\frac{N_{D}\left(N_{A 1}+N_{A 2}-N_{D}\right)\left[C_{p S} N_{S}+C_{p A}\left(N_{D}-N_{A 1}\right)\right]^{2} N_{v}}{\left(N_{A 1}+N_{A 2}\right)\left(N_{D}-N_{A 1}\right)\left(C_{p S} N_{S}+C_{p A} N_{D}\right) g} \text {. }
$$

Note that $\eta_{1}$ and $\eta_{2}$ start decreasing simultaneously during the transition from region II to region III. Thus, the expression (26) for $T_{1}$ is valid for both $\eta_{1}$ and $\eta_{2}$. According to Eq. (26), the characteristic temperature $T_{1}$ increases with increasing excitation intensity, as demonstrated in Fig. 5.

The value of the step for PL associated with the deeper acceptor during the transition from region II to region III, $R_{1}$, can be expressed from Eqs. (20) and (25):

$$
R_{1}=\frac{\eta_{2, I I}}{\eta_{2, I I I}}=\frac{N_{A 2} N_{D}\left[C_{p S} N_{S}+C_{p A}\left(N_{D}-N_{A 1}\right)\right]}{\left(N_{A 1}+N_{A 2}\right)\left(N_{D}-N_{A 1}\right)\left(C_{p S} N_{S}+C_{p A} N_{D}\right)} .
$$

As can be seen from Eq. (28), the depth of the first step is very sensitive to the value of $\left(N_{D}-N_{A 1}\right)$. It is close to unity (i.e., no step) when $N_{A 1} \ll N_{D}$, and it increases substantially when $N_{A 1}$ approaches $N_{D}$. Figure 9 shows how the first step in the quenching of PL via the deeper acceptor depends on the concentration of the shallow acceptor when other parameters are fixed. Note that Eq. (28) is not valid for the case when $N_{A 1} \geqslant N_{D}$, although numerical calculations using Eqs. (2)-(8) show that the first step continues to increase when $N_{A 1}$ exceeds $N_{D}$ (Fig. 9). Finally, when $N_{A 1} \geqslant N_{D}+N_{S}$, we observe an abrupt step at $T \approx T_{1}$, and no other steps can be found in the temperature dependence of PL from both the shallow and deeper acceptors.

\section{Second step in thermal quenching of photoluminescence}

At higher temperatures, emission of holes from the deeper acceptor to the valence band becomes substantial (transition from region III to region IV begins at $T \approx 150 \mathrm{~K}$ ). In this transition, a very abrupt quenching is observed for PL from both the shallow and deeper acceptors at temperature close to $T_{2}$. On the low-temperature side of this abrupt transition, variables $N_{A 1}^{-}, N_{A 2}^{-}, N_{S}^{0}$, and $N_{D}^{+}$remain constant and equal

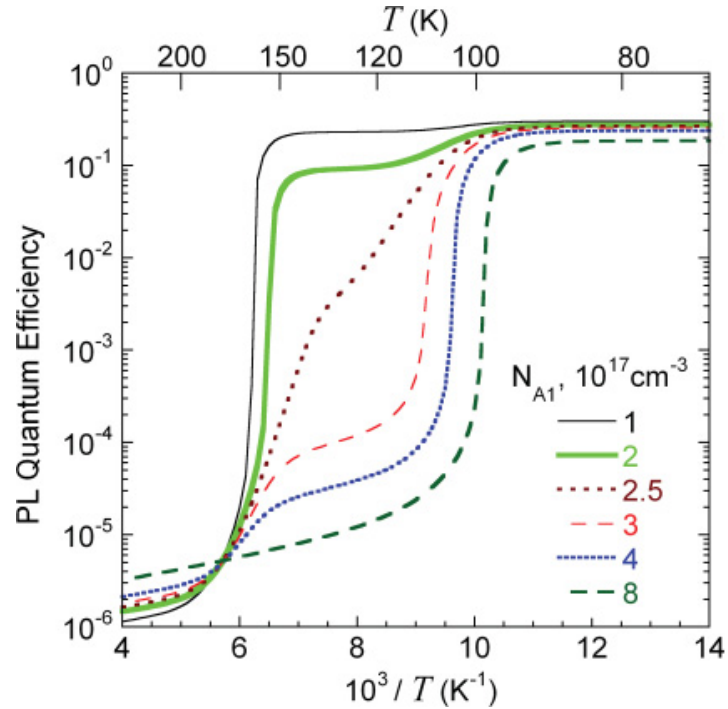

FIG. 9. (Color online) Temperature dependence of the quantum efficiency $\eta_{2}$ of PL associated with the deeper acceptor $A_{2}$ for different concentrations of the shallow acceptor $A_{1}$. The values of $N_{A 1}$ are shown on the graph. Other parameters are listed in Table I as Set 2. $G=10^{20} \mathrm{~cm}^{-3} \mathrm{~s}^{-1}$.

to their values in region III: $N_{A 1}^{-} \approx N_{A 1}, N_{A 2}^{-} \approx N_{D}-N_{A 1}$, $N_{S}^{0} \approx N_{S}$, and $N_{D}^{+} \approx N_{D}$. The processes occurring during the transition from region III to $T \approx T_{2}$ and the analytical formulas describing them are very similar to those at temperatures preceding the abrupt quenching of PL in a high-resistivity $p$-type semiconductor with only one type of acceptor. ${ }^{2}$ In particular, the quantum efficiency of PL associated with the deeper acceptor in the transition from region III to $T_{2}$ varies as

$$
\eta_{2, I I I \rightarrow T_{2}}=\eta_{2, I I I}\left[1-\xi \exp \left(-E_{A 2} / k T\right)\right]
$$

with

$$
\xi=\frac{C_{p S} N_{S}\left(N_{A 1}+N_{A 2}-N_{D}\right) N_{v}}{\left(N_{D}-N_{A 1}\right) g G} .
$$

The abrupt thermal quenching of PL occurs at

$$
T_{2} \approx T_{2}^{*}=\frac{E_{A 2}}{k \ln \left(B_{2} / G\right)},
$$

where

$$
B_{2}=\frac{C_{p S} N_{S}\left(N_{A 1}+N_{A 2}-N_{D}\right) N_{v}}{\left(N_{D}-N_{A 1}\right) g} .
$$

Note that $T_{2}^{*}$, initially introduced in Ref. 2 as $T^{*}$ and generalized here for the case of two acceptors, just slightly differs from $T_{2}$ defined in a simpler way in Sec. III.

The quantum efficiency of PL via the shallow acceptor also abruptly drops at $T_{2}$ (Fig. 8). According to Eqs. (26) and (31), $T_{1}$ and $T_{2}$ increase with increasing excitation intensity, and the regions of PL quenching shift to higher temperatures (Fig. 5). The value of $10^{3} / T_{1}$ shifts faster than $10^{3} / T_{2}$ with increasing excitation intensity. In other words, the slope of the dependence of $10^{3} / T_{1}$ on $\ln G$ is larger than that for $10^{3} / T_{2}$ by a factor of $E_{A 2} / E_{A 1}$. Equation (32) is valid only for $N_{A 1}<N_{D}$. When $N_{D}+N_{S}>N_{A 1}>N_{D}$, the second step in the quenching of PL from a deep acceptor is barely noticeable and its position is 
close to the position defined with Eqs. (31) and (32) in which $\left(N_{D}-N_{A 1}\right)$ should be replaced with $N_{D}$. With further increase in $N_{A 1}$, when $N_{A 1}>N_{D}+N_{S}$, the second step disappears and only an abrupt step at $T \approx T_{1}$ is observed, as can be seen from Fig. 9.

After the abrupt drop (region IV), the quantum efficiency of PL via the shallow acceptor is

$$
\begin{aligned}
\eta_{1, I V}= & \left(C_{n A}+C_{D A} \frac{g N_{D}}{N_{c}} e^{E_{D} / k T}\right) \\
& \times \frac{N_{A 1}\left(N_{A 1}+N_{A 2}-N_{S}-N_{D}\right)}{C_{n S} N_{S}\left(N_{S}+N_{D}-N_{A 1}\right)} \exp \left(\frac{E_{A 1}-E_{A 2}}{k T}\right) .
\end{aligned}
$$

It increases with increasing temperature but remains small as compared to $\eta_{2, I V}$. The quantum efficiency of PL associated with the deeper acceptor, after the abrupt drop, saturates in region IV at

$\eta_{2, I V}=\left(C_{n A}+C_{D A} \frac{g N_{D}}{N_{c}} e^{E_{D} / k T}\right) \frac{\left(N_{A 1}+N_{A 2}-N_{S}-N_{D}\right)}{C_{n S} N_{S}}$.

The value of the step in $\eta_{2}$ during the transition from region III to region IV is defined as $R_{2}=\eta_{2, I I I} / \eta_{2, I V}\left(T_{2}^{*}\right)$. The value of the step at $T \approx T_{2}^{*}$ is almost identical to the step in the case of one acceptor, ${ }^{2}$ with the difference that $N_{A}$ should be replaced with $N_{A 1}+N_{A 2}$.

When a semiconductor is high-resistivity $n$-type $\left(N_{D}+\right.$ $N_{S}>N_{A 1}+N_{A 2}>N_{D}>N_{A 1}$ ), two steps are observed, and the drops in PL intensity occur at about the same temperatures as in the $p$-type material. However, as in the case of one acceptor, ${ }^{2}$ after the abrupt quenching at $T \approx$ $T_{2}$, the PL intensities related to both acceptors continue to decrease exponentially: $\eta_{1, I V} \propto \exp \left[\left(E_{A 1}+E_{D}\right) / k T\right]$ and $\eta_{2, I V} \propto \exp \left[\left(E_{A 2}+E_{D}\right) / k T\right]$ (Fig. 10).

\section{DISCUSSION}

\section{A. Comparison with experiment}

At low temperatures (up to $\sim 70 \mathrm{~K}$ ), we can distinguish the contributions of the $e A$ and DAP components of the UVL band (Fig. 11). The quantum efficiency of the $e A$ component increases exponentially with decreasing $10^{3} / T$. An activation energy of this increase is close to $5 \mathrm{meV}$, which is smaller than the separation between the $e A$ and DAP peaks $[20 \mathrm{meV}$, as can be seen in Fig. 2(c)] or the ionization energy of shallow donors in GaN (about $30 \mathrm{meV}$ ). ${ }^{1}$ This discrepancy can be attributed to a relatively high concentration of shallow donors and $\mathrm{Zn}_{\mathrm{Ga}}$ acceptors in $\mathrm{Zn}$-doped $\mathrm{GaN}$. The Coulomb interaction between donors and acceptors shifts the donor level in the direction of the conduction band, with the value of the shift depending on the distance between a particular donor and the nearest acceptor. Moreover, interaction between shallow donors "broadens" the shallow donor level. Due to a random distribution of the DAP separations, there is a distribution of the donor energy, with the mean value closer to the conduction band than it is in a semiconductor

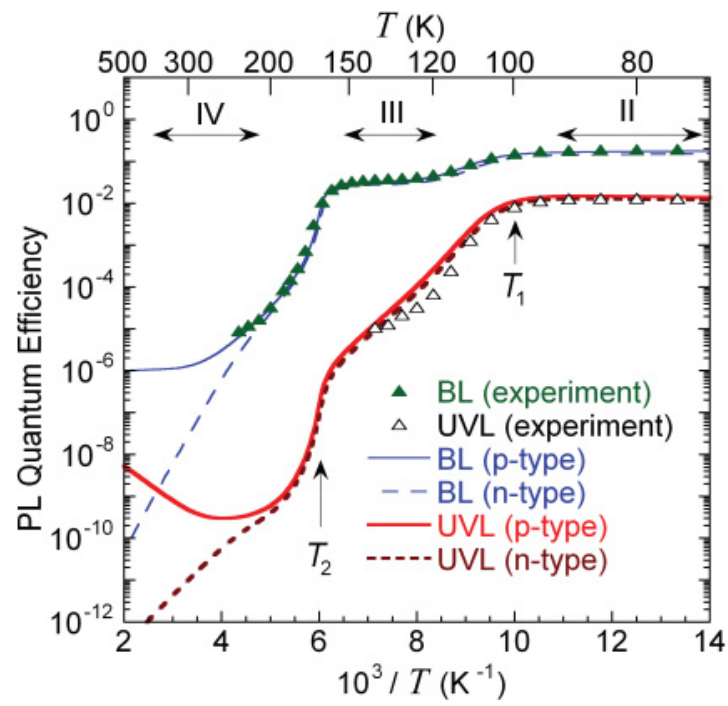

FIG. 10. (Color online) Temperature dependence of PL quantum efficiency for the BL and UVL bands at $P_{\text {exc }}=0.0015 \mathrm{~W} / \mathrm{cm}^{2}$ $\left(G=2.4 \times 10^{20} \mathrm{~cm}^{-3} \mathrm{~s}^{-1}\right)$. Points are experimental data. Lines are numerical calculations using Eqs. (2)-(8) with $N_{S}=2.5 \times 10^{17} \mathrm{~cm}^{-3}$ ( $p$ type) and $N_{S}=3.0 \times 10^{17} \mathrm{~cm}^{-3}$ ( $n$ type) and other parameters from Set 1 in Table I. Roman numerals on top indicate temperature regions discussed in the text.

with a very low concentration of impurities. In the optical experiments, the separation between the $e A$ and DAP lines corresponds to the mean distance between the donor levels and the conduction band. However, in electrical effects (such as thermal emission of electrons to the conduction band) the most shallow levels will contribute more because at a given

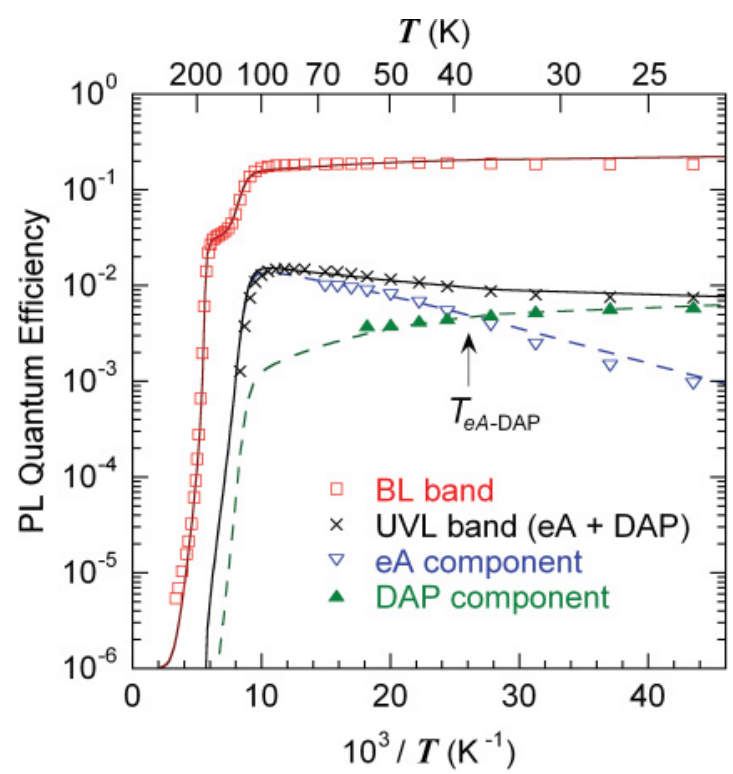

FIG. 11. (Color online) Temperature dependence of PL quantum efficiency for the PL and UVL bands at $P_{\text {exc }}=0.0105 \mathrm{~W} / \mathrm{cm}^{2}$. Points are experimental data. Lines are numerical calculations using Eqs. (2)-(8) with $G=1.68 \times 10^{21} \mathrm{~cm}^{-3} \mathrm{~s}^{-1}$ and other parameters from Set 1 in Table I. Dashed curves show the calculated data for the $e A$ and DAP components of the UVL band, while solid curves show the combined intensity from $e A$ and DAP transitions for the $\mathrm{BL}$ and UVL bands. 
temperature the rate of electron emission from shallow donors increases exponentially with decreasing the distance between the donor level and the conduction band minimum. For this reason, the value of $E_{D}=5 \mathrm{meV}$ (which is needed to explain the temperature dependence of the $e A$ component of the UVL band) is considered as reasonable and was used in the fitting of all other experimental data as well.

As was shown in Sec. III, the $e A$ component of the UVL band is weaker than the DAP component at very low temperatures, but it increases with temperature and eventually becomes stronger. The characteristic temperature $T_{e A-\mathrm{DAP}}$, at which intensities of these two components are equal, corresponds to the condition $C_{n A 1} N_{A 1}^{0} n=C_{D A 1} N_{D}^{0} N_{A 1}^{0}$, which provides some information about the capture coefficients $C_{n A 1}$ and $C_{D A 1}$ for the shallow acceptor. When the thermal emission of electrons to the conduction band is substantial (region II), $C_{n D} N_{D}^{+} n \approx$ $Q_{D} N_{D}^{0}$, and the following expression can be derived:

$$
C_{n A 1} \frac{N_{c}}{g N_{D}} \exp \left(-\frac{E_{D}}{k T_{e A-\mathrm{DAP}}}\right)=C_{D A 1} .
$$

This allows us to find an approximate relation between $C_{n A 1}$ and $C_{D A 1}$ with the assumption that $N_{D} \approx 10^{17} \mathrm{~cm}^{-3}$. The quantum efficiencies of the UVL and BL bands at low temperatures (regions I and II) are very sensitive to the relative values of the hole-capture coefficients for radiative and nonradiative defects. This is because at low temperatures the PL intensities are governed by a competition between different recombination channels for photogenerated holes. Since coefficients $C_{p A 1}$ and $C_{p A 2}$ are known from temperature dependencies of PL in conductive $n$-type samples having the $\mathrm{Zn}_{\mathrm{Ga}}$ and shallow acceptors, ${ }^{1}$ coefficient $C_{p S}$ can be roughly estimated from the low-temperature PL quantum efficiencies. Furthermore, at low temperatures, the PL intensities are insensitive to variation of coefficients $C_{n D}$ and $C_{n S}$, provided that $C_{n D}<C_{n S}$. When $C_{n D} \gg C_{n S}$, the quantum efficiency of PL associated with two acceptors approaches unity, which contradicts the experimental data.

The value of the first step in the thermal quenching of the BL band, $R_{1}$, is very sensitive to the difference between the concentrations of the shallow donor and shallow acceptor [see Eq. (28) and Fig. 9]. We do not see the first step in the thermal quenching of the BL band in most of the $\mathrm{Zn}$-doped GaN samples, which implies that the concentration of the shallow acceptor in these samples is much lower than the concentration of the shallow donor. A significant stepwise drop of the BL band intensity at $T \approx T_{1}$ for sample s560 indicates that $N_{A 1} \approx N_{D}$ in this sample. It is expected that $N_{D} \approx 10^{17}-10^{18} \mathrm{~cm}^{-3}$ due to contamination with oxygen, and this value should not differ much from sample to sample. However, the quantum efficiency of the UVL band varies significantly in different $\mathrm{Zn}$-doped GaN samples; see Table I in Ref. 2. Assuming that $N_{D}=10^{17} \mathrm{~cm}^{-3}$, we can estimate that $N_{A 1} \approx 10^{17} \mathrm{~cm}^{-3}$ in sample s560, but $N_{A 1} \approx 10^{14}$ $10^{15} \mathrm{~cm}^{-3}$ in samples with an equally strong BL band and a very weak UVL band (samples ap269, 1393, and 1394).

\section{B. Parameters of the model}

Some parameters of the model applied to the case of high-resistivity GaN:Zn are known from independent studies.
In particular, the hole-capture coefficient for the $\mathrm{Zn}_{\mathrm{Ga}}$ acceptor was estimated as $C_{p A 2}=7 \times 10^{-7} \mathrm{~cm}^{3} / \mathrm{s}$ from fitting the temperature dependence of the BL band intensity in $n$-type GaN. ${ }^{13}$ The hole-capture coefficient for the shallow acceptor was previously estimated as $C_{p A 1}=10^{-6} \mathrm{~cm}^{3} / \mathrm{s}$ from the thermal quenching of the UVL band in undoped GaN grown by the MOCVD method. ${ }^{7}$ However, for undoped GaN samples grown by the MBE method, we obtained the value of $C_{p A 1}=$ $7 \times 10^{-8} \mathrm{~cm}^{3} / \mathrm{s}$ from the thermal quenching of the UVL band. It is possible that different types of shallow acceptors are responsible for the UVL band in these two studies.

The electron-capture coefficients for the shallow and $\mathrm{Zn}_{\mathrm{Ga}}$ acceptors in the MOCVD-grown GaN were estimated as $C_{n A 1}=4 \times 10^{-12} \mathrm{~cm}^{3} / \mathrm{s}$ and $C_{n A 2}=4 \times 10^{-13} \mathrm{~cm}^{3} / \mathrm{s}$, respectively, by using information about the lifetime of the UVL and BL bands obtained from time-resolved PL experiments. ${ }^{14}$ A slightly different value of $C_{n A 1}=2 \times 10^{-12} \mathrm{~cm}^{3} / \mathrm{s}$ has been reported for the shallow acceptor in undoped GaN grown by the HVPE method. ${ }^{15}$

Ionization energies of the shallow and $\mathrm{Zn}_{\mathrm{Ga}}$ acceptors are known from PL quenching in $n$-type GaN. Concentrations of the $\mathrm{Zn}_{\mathrm{Ga}}$ acceptor and $\mathrm{O}_{\mathrm{N}}$ donor are expected to be in the range of $10^{17}-10^{18} \mathrm{~cm}^{-3}$ in $\mathrm{Zn}$-doped GaN, as discussed in Ref. 2. The absolute internal quantum efficiency of all PL bands was estimated by comparison of integrated PL intensities with PL intensity from a calibrated standard, as discussed in Ref. 2.

Note that excessive constraint of model parameters makes it difficult to obtain a good fit with experimental data, because there are many characteristic features in the experimental dependencies. For example, we reproduce the quantum efficiency of two PL bands in different temperature regions and different excitation intensities, values of $T_{1}$ and $T_{2}$ and their dependence on excitation intensity, and relative intensities of the $e A$ and DAP components of the UVL band and their temperature dependencies. In order to get a better fit, we slightly varied the parameters that are expected to be known from other experiments (see Set 1 in Table I). In any case, we do not claim that the parameters found from the fit are unique or very accurate. They are rather used as an illustration of the fact that a set of reasonable parameters within a simple phenomenological model explains well many features of unusual temperature dependencies of PL.

\section{Shallow acceptor in GaN}

A two-step thermal quenching of the BL band was observed only in one sample (s560) out of eight high-resistivity GaN samples doped with $\mathrm{Zn}$. For other samples, only one step of abrupt quenching of the BL band was observed which was explained in a model including a $\mathrm{Zn}_{\mathrm{Ga}}$ acceptor, a shallow donor $\mathrm{O}_{\mathrm{N}}$, and an unidentified nonradiative deep donor. ${ }^{2}$ The two-step quenching, reported here, is naturally explained within the same model when a second (shallow) acceptor is included with a concentration lower than the concentrations of any other species, as we demonstrated in Sec. IV. The origin of the shallow acceptor in $\mathrm{Zn}$-doped $\mathrm{GaN}$ is unknown. It is known that $\mathrm{Mg}_{\mathrm{Ga}}$ is a shallow acceptor with ionization energy in the range from 0.15 to $0.2 \mathrm{eV}$ when $\mathrm{GaN}$ is doped with $\mathrm{Mg}$. ${ }^{1}$ For undoped $n$-type GaN, the UVL band (with its $e A$ and DAP components) is attributed to a shallow acceptor of unknown 
origin. The shape and position of the UVL band is about the same in Mg-doped and undoped GaN. The ionization energy of this shallow acceptor is close to $0.2 \mathrm{eV}$ in all cases. Several suggestions about its origin were proposed, among which the ones suggested in Refs. 16-18 are most worthy of notice.

\section{CONCLUSIONS}

We observed a two-step thermal quenching of the BL band in $\mathrm{Zn}$-doped GaN. This is the first observation of abrupt and tunable two-step quenching of PL in semiconductors. The effect is explained in a phenomenological model, which includes four types of defects: a shallow donor, a shallow acceptor, a deeper acceptor, and a deep nonradiative donor. The first step in the thermal quenching of the BL band (at $T \approx 100 \mathrm{~K}$ ) is caused by the emission of holes from the shallow acceptor to the valence band and their recapture by other defects, primarily by the deeper $\mathrm{Zn}_{\mathrm{Ga}}$ acceptor. The second step in the quenching of the BL band is caused by emission of holes from the $\mathrm{Zn}_{\mathrm{Ga}}$ acceptor to the valence band and their recapture by the nonradiative deep donor. Positions of both steps are tunable; namely, the characteristic temperatures of the quenching shift to higher temperatures with increasing excitation intensity. The slope of the dependence of the inverse characteristic temperature on the logarithm of the excitation intensity reveals the ionization energy of the acceptor responsible for the thermal quenching. *mreshchi@vcu.edu

${ }^{1}$ M. A. Reshchikov and H. Morkoç, J. Appl. Phys. 97, 061301 (2005).

${ }^{2}$ M. A. Reshchikov, A. A. Kvasov, M. F. Bishop, T. McMullen, A. Usikov, V. Soukhoveev, and V. A. Dmitriev, Phys. Rev. B 84, 075212 (2011).

${ }^{3}$ H. A. Klasens, J. Phys. Chem. Solids 9, 185 (1959).

${ }^{4}$ K. Maeda, J. Phys. Chem. Solids 26, 595 (1965).

${ }^{5}$ K. Maeda, J. Phys. Chem. Solids 26, 1419 (1965).

${ }^{6}$ M. A. Reshchikov, X. Gu, J. Nause, and H. Morkoç, Proc. Mater. Res. Soc. Symp. 892, FF23-11 (2006).

${ }^{7}$ M. A. Reshchikov and R. Y. Korotkov, Phys. Rev. B 64, 115205 (2001).

${ }^{8}$ M. A. Reshchikov, A. G. Willyard, A. Behrends, A. Bakin, and A. Waag, Appl. Phys. Lett. 99, 171107 (2011).

${ }^{9}$ A. M. Stoneham, Theory of Defects in Solids (Clarendon Press, Oxford, 2001).

${ }^{10}$ C. G. Van de Walle and J. Neugebauer, J. Appl. Phys. 95, 3851 (2004).
${ }^{11}$ W. Shockley and W. T. Read, Phys. Rev. 87, 835 (1952).

${ }^{12}$ V. N. Abakumov, V. I. Perel, and I. N. Yassievich, Nonradiative Recombinations in Semiconductors (Elsevier, Amsterdam, 1991).

${ }^{13}$ M. A. Reshchikov, M. A. Foussekis, J. D. McNamara, A. Behrends, A. Bakin, and A. Waag, J. Appl. Phys. 111, 073106 (2012).

${ }^{14}$ R. Y. Korotkov, M. A. Reshchikov, and B. W. Wessels, Physica B 325, 1 (2003).

${ }^{15}$ M. A. Reshchikov, S. S. Park, K. Y. Lee, and H. Morkoç, Physica B 340-342, 448 (2003).

${ }^{16}$ B. Monemar, P. P. Paskov, F. Tuomisto, K. Saarinen, M. Iwaya, S. Kamiyama, H. Amano, I. Akasaki, and S. Kimura, Physica B 376-377, 440 (2006).

${ }^{17}$ B. Monemar, P. P. Paskov, J. P. Bergman, M. Iwaya, S. Kamiyama, H. Amano, and I. Akasaki, Physica B 376-377, 460 (2006).

${ }^{18}$ T. Paskova, B. Armaudov, P. P. Paskov, E. M. Goldys, S. Hautakangas, K. Saarinen, U. Södervall, and B. Monemar, J. Appl. Phys. 98, 033508 (2005). 